\title{
Special issue on NETYS selected papers
}

\author{
Vincent Gramoli $^{1}$ - Rachid Guerraoui ${ }^{2}$
}

Published online: 26 July 2015

(C) Springer-Verlag Wien 2015

\section{Introduction}

Modern scalable systems tend to become more and more distributed, relying on networked communications to distribute tasks among multiple cores, commodity computers, or datacenter servers. These communication media span different scales from network-on-chip, to wired networked and to wireless networks. The communications are specified through algorithms that have to be resilient to failures, security threats and attacks. This special issue aims at gathering new ideas related to the context of networked systems and comprises a selection of the best papers presented at the International Conference on Networked Systems in 2013, NETYS 2013.

\section{Selected papers}

The selected papers tackle the important problems related to the performance and the privacy of message-based and shared memory communications.

In the paper entitled "Improving resource location with locally precomputed partial random walks" the authors propose a way to retrieve resources efficiently in an unstructured distributed network by pre-computing partial random walks before a lookup request arrives and by exploiting Bloom filters to save storage space while indicating whether a particular walk leads to the targeted resource.

Vincent Gramoli

vincent.gramoli@sydney.edu.au

Rachid Guerraoui

rachid.guerraoui@epfl.ch

1 NICTA and University of Sydney, Sydney, Australia

2 EPFL, Lausanne, Switzerland 
The authors of the paper "A distributed protocol for privacy preserving aggregation with non-permanent participants" present a privacy-preserving aggregation technique that does not rely on cryptography. It enables the computation of aggregation functions that can be expressed as Abelian group.

In the paper "Airtime pingpong effect characterization in IEEE 802.11s wireless mesh networks", the authors identified a ping-pong problem related to the rate control of IEEE $802.11 \mathrm{~s}$ where links oscillate between overloaded and non-overloaded states.

The authors of "Identifying the optimal level of parallelism in transactional memory applications" present a way to adapt dynamically the level of parallelism that maximizes the throughput of transactions in shared memory and distributed transactional memory (TM) applications.

Finally, the authors of the paper "FreeRec: an Anonymous and Distributed Personalization Architecture" exploit onion routing to implement a distributed protocol that routes information to a node while anonymizing the source. This idea finds practical applications in distributed voting systems that preserve anonymity.

Acknowledgments We would like to thank all the authors of NETYS 2013 as well as the reviewers of the selected papers of this special issue who thoroughly evaluated their quality through a rigorous process. 